\title{
Patterns of synuclein expression throughout lens development
}

\author{
Irina Surgucheva ${ }^{1,2}$, Belinda MacMahon ${ }^{3}$, Andrei Surguchov $^{1,2^{*}}$ \\ ${ }^{1}$ Retinal Biology Research Laboratory, Veterans Administration Medical Center, Kansas City, USA; *Corresponding Author: \\ asurguchov@kumc.edu \\ ${ }^{2} \mathrm{MO}$ and Department of Neurology, Kansas University Medical Center, Kansas City, USA \\ ${ }^{3}$ Washington University, St. Louis, USA
}

Received 20 December 2009; revised 4 February 2010; accepted 25 February 2010.

\begin{abstract}
Synucleins belong to a family of small soluble proteins with chaperonic activity implicated in human diseases, but their normal function is not completely understood. Expression of $\alpha, \beta$ and $\gamma$-synucleins was analyzed in rat and human lens on different stages of development. No significant expression of $\alpha$ - and $\beta$-synucleins were found, whereas $\gamma$-synuclein was expressed in both species only on early stages of lens development. Examination of $\gamma$-synuclein upstream region demonstrated the similarity in its organization with promoter regions of crystallins and heat shock protein's genes. $\gamma$-Synuclein upstream region contains motives identical or similar to regulatory cis-elements in their promoters including binding sites for Pax6 and Sox. These results suggest that $\gamma$-synuclein plays a role in distinct temporal events in lens development, presumably acting as a specific chaperone.
\end{abstract}

Keywords: Lens; Crystallins; Synucleins; Promoter; Chaperones; Heat Shock Proteins

\section{INTRODUCTION}

The heat shock proteins (Hsps) are members of a gene family encoding molecular chaperones: the cytoplasmic mediators or protein folding. Chaperones in addition to their assistance in protein folding/unfolding fulfill multiple housekeeping functions, such as transport across membranes, participation in protein degradation [1] and modulation of the cytoskeleton [2].

Several reports demonstrated an important role of chaperones in embryonic development. For example, Hsp47 is a chaperone essential for early mammalian embryogenesis [3], members of the Hsp70 family control germ cell meiosis in mice [4] and Hsp70 is required for embryonic lens formation [5].
Synucleins are a family of small proteins with chaperonic properties $[6,7]$ consisting of three members: $\alpha-$, $\beta$-, and $\gamma$-synuclein (reviewed in [8]). Synucleins are abundant soluble proteins found primarily in nervous systems the functions of which are not completely understood. All synuclein members share a common structural design, with the first two thirds of the sequence organized within a series of amphipathic domains that may facilitate membrane binding. All three synucleins consist of a highly conserved $\mathrm{N}$-terminal domain that includes a variable number of 11 amino acid repeats, and a less conserved $\mathrm{C}$-terminal domain with a relatively high content of acidic amino acids. Synucleins are naturally unfolded proteins and they change their conformation dramatically upon lipid binding $[9,10]$.

The functional role of all three synucleins in the development and maturation of neurons has been demonstrated by several groups. $\alpha$-Synuclein has an important function in prenatal development and in the formation of the nervous system [11]. It is expressed in neurons very early in human gestation, starts disappearing in early childhood and reappear in the adult neurons [12]. $\gamma$-Synuclein has a distinct pattern of expression in the developing nervous system [13]. In eye tissues synucleins are expressed in neural retina and optic nerve [14], however, their presence in other ocular tissues was not thoroughly investigated.

We decided to examine whether members of the synuclein family are expressed in the course of lens development. The lens is an ocular tissue in which all cells are derived by the proliferation and differentiation of lens epithelial cells expressing numerous molecular chaperones. Several chaperones, for example, $\alpha$-crystallins, are required for specific developmental events and are expressed during a short period of embryonic lens development [5,15-17]. We found significant developmental alterations in the expression of $\gamma$-synuclein in rat and human lens. The highest level of $\gamma$-synuclein expression in the lens was found in the embryonic steps, at early post natal steps its expression subsides and it is reduced considerably in the mature eye. The upstream region of 
$\gamma$-synuclein gene contains nucleotide sequences similar to regulatory elements in crystallin genes and binding sites for Pax6 and other transcription factors controlling eye development.

\section{EXPERIMENTAL PROCEDURES}

\subsection{Materials and Methods}

Human fetal eyes were a generous gift of Dr. Rosario Hernandez (Department of Ophthalmology, Washington University School of Medicine, St. Louis, MO) received through the International Institute for the Advancement of Science (Exton, PA) and the Anatomical Gift Foundation (Laurel, MD). The samples with gestational age of 42 days, 44 days, 57 days and 20 weeks were used. The use of these samples was approved by Washington University Human Study commission. The eyes were fixed immediately after enucleation in $10 \%$ buffered formaldehyde for at least 6 hours.

Samples of rat's eyes were a kind gift of Dr. David Beebe (Department of Ophthalmology, Washington University School of Medicine, St. Louis, MO). Rats were cared for and handled in accordance with the ARVO Statement for the Use of Animals in Ophthalmic and Vision Research and were approved by the Washington University Animal Studies Committee.

\section{Antibodies}

Antibody against human $\gamma$-synuclein was raised in rabbit against the most unique portion of the protein and used as described earlier at dilution 1:2,500 [14]. For rat eye staining the dilution was 1:1,500. $\alpha$ - and $\beta$-synuclein antibody was purchased from Chemicon International Inc. (Temecula, CA) diluted 1:1,500 and 1:2,000, respectively. The specificity of antibodies was checked by Western blotting.

\subsection{Immunohistochemical Staining}

Eyes were fixed in paraformaldehyde/PBS, pH 7.4 at $40^{\circ} \mathrm{C}$. From 8 to 12 sections were prepared and analyzed. After ennucleation eyes were washed in PBS containing $0.1 \%$ glycine and processed for paraffin embedding. Slices of $5 \mu \mathrm{m}$ were cut and placed on silane-coated slides. Before immunostaining, slides were deparaffinized and incubated for $1 \mathrm{~h}$ in PBS/glycine at RT to reduce non-specific binding. Slides were preincubated with $5 \%$ milk for $30 \mathrm{~min}$, rinsed and incubated with primary antibody for $30 \mathrm{~min}$.

Biothynilated secondary antibody was placed on the sections and incubated for $30 \mathrm{~min}$, washed with PBS, and reacted with streptavidin-peroxidase conjugate (Vector Laboratories) for $30 \mathrm{~min}$. The bound antibodyperoxidase complexes on the sections were visualized using a 3,3'-diaminobenzidine tetrahydrochloride (DAB) substrate solution consisting of $1.5 \mathrm{mg}$ of DAB and $50 \mu \mathrm{l}$ of $30 \%$ hydrogen peroxide in $10 \mathrm{ml}$ of $100 \mathrm{mM}$ Tris- $\mathrm{HCl}$, $\mathrm{pH}=7.6$. The sections were incubated in the darkness until brown staining appeared, washed in PBS, counterstained with hematoxylin, dehydrated, and coverslipped with Permount. Control sections were run in parallel, omitting the primary antibody. Other details of immunochemical staining are described previously [14].

\subsection{Multiple Sequence Alignment}

Multiple sequence alignment was performed using computer program Clustal W [18], pairwise alignment by Smith-Waterman full-length alignments between two sequences.

\section{Results}

To analyze the pattern of synuclein expression in lens tissues as a function of development, ocular tissues from rats from three age groups were used: day 18 embryos (E18), day 4 newborn animals (P4) and 10 month old rats (Figure 1). Human embryonic tissues at days 42, 44, 57 and 20 weeks were analyzed (Figure 2).

Results presented on Figure 1, demonstrate only slight aged-dependent variations in $\alpha$-synuclein $(\mathrm{a}, \mathrm{d}, \mathrm{g}$ ) and $\beta$-synuclein $(\mathrm{b}, \mathrm{e}, \mathrm{h}$ ) expression in the rat's eye and significant developmental changes for $\gamma$-synuclein expression $(\mathrm{c}, \mathrm{f}, \mathrm{i})$.

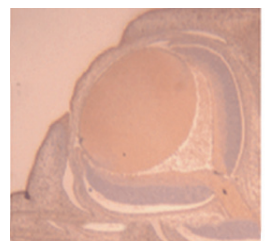

(a)

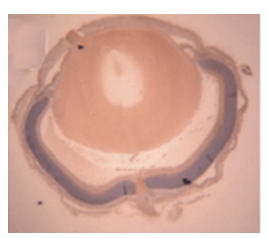

(d)

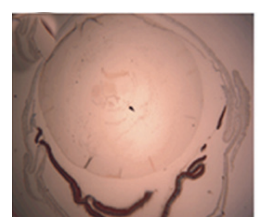

(g)

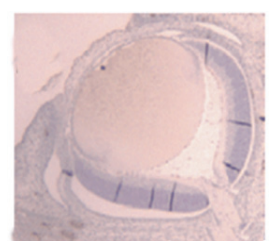

(b)

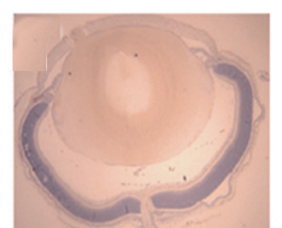

(e)

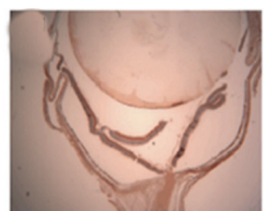

(h)

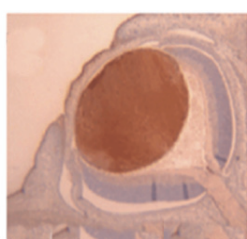

(c)

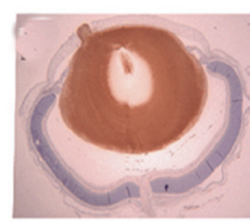

(f)

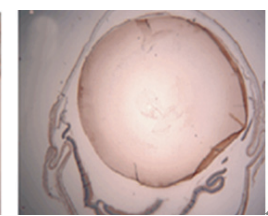

(i)
Figure 1. Immunohistochemical detection of. synuclein reactivity in various parts of a rat eye on different steps of development. Top row (a-c) - 18 days embryos; middle row (d-f) -4 day old rats; bottom row (g-i) - 10 months old rats. Left panel (a, d, g) $-\alpha$-synuclein; middle panel (b, e, h) $-\beta$-synuclein; right row $(\mathrm{c}, \mathrm{f}, \mathrm{i})-\gamma$-synuclein. 


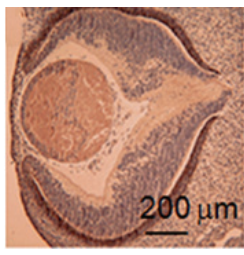

(a)

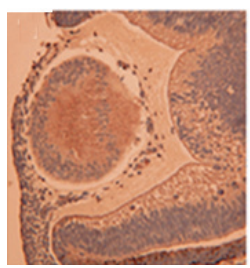

(d)

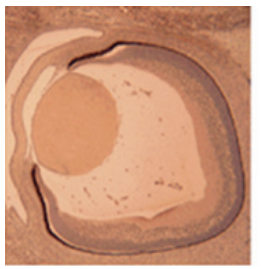

(g)

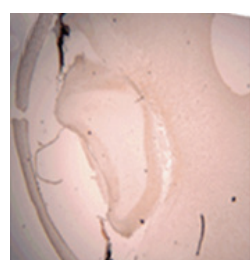

(j)

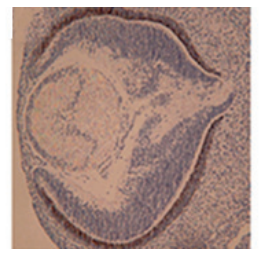

(b)

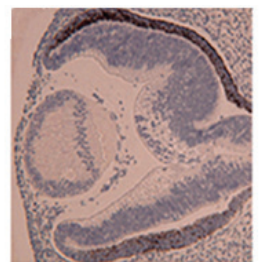

(e)

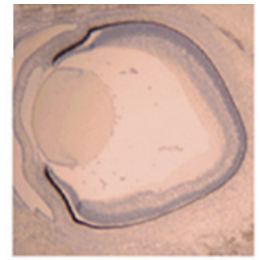

(h)

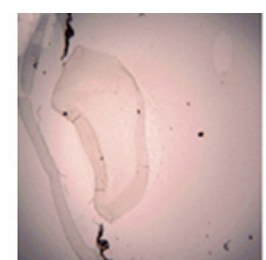

(k)

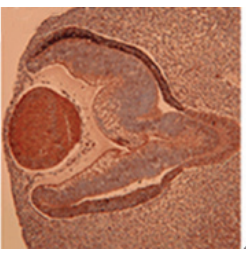

(c)

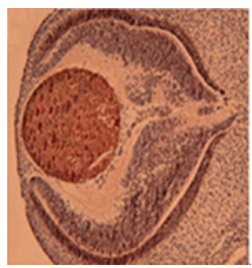

(f)

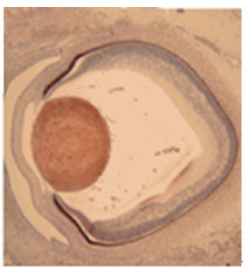

(i)

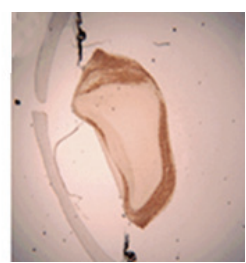

(1)
Figure 2. Immunohistochemical detection of synucleins in various parts of the human eye on different steps of development. Top row: 42 days, second row -48 days, third row -57 days, bottom row -20 weeks. Left column - $\alpha$-synuclein, middle column - $\beta$-synuclein, right column - $\gamma$-synuclein.

The highest level of $\gamma$-synuclein expression is in the $\mathrm{E} 18$ lens, at $\mathrm{P} 4$ the immunoreactivity becomes less intensive in the periphery of the lens and practically disappears in the central region. The lens from adult animal was not stained, except faintly in the periphery (Figure 1(i)).

In E18 rat evidence of $\gamma$-synuclein expression was detectable in the majority of both the lens epithelium and differentiating lens fibers (Figure 1(c)). In postnatal 4 days rats $\gamma$-synuclein nuclear level was decreased (Figure 1(f)) and in 10 month old rat's lens $\gamma$-synuclein is not detectable (Figure 1(i)).

Staining of human embryonic ocular tissues revealed basically the same temporarily alterations in the pattern of expression (Figure 2). Weak $\alpha$-synuclein staining is present in the nuclear area of the lens at early stages of development (42 and 44 days), reduced by day 57 and disappears by 20 weeks. $\beta$-Synuclein is not expressed in the lens at any developmental stage (Figures 2(b), (e), (h) and (k)). $\gamma$-Synuclein immunoreactivity is detectable in the whole lens on day 42 (Figure 2(c)), and disappears gradually first from the nuclear area of the lens and later from the whole lens (Figures 2(f), (i) and (I)).

Then we asked whether these temporary expression of $\gamma$-synuclein may be due to the transcriptional regulation of its gene in a similar way as the regulation of crystallin genes implicated in lens development. So we searched the upstream region of $\gamma$-synuclein gene for cisregulatory elements similar to that controlling the developmental expression of crystallins and other lens proteins.

We found that $\gamma$-synuclein and crystallins have similar cis-acting regulatory elements in the upstream regions of their genes. For example, palindromic sequence -102 GGGAGATCCC -93 in $\gamma$-synuclein gene is similar to the regulatory elements downstream from -110 in the mouse and -109 in the chicken $\alpha \mathrm{A}$-crystallin gene (Figure 3) [19-21]. This palindrome has a key role in the activity of the $\alpha \mathrm{A}$-crystallin promoter and the regulated expression of this gene is indispensable for lens development. The importance of this palindromic element was also proven in experiments with cultured mouse lens cells [19]. Another sequence in $\gamma$-synuclein upstream region is decamer -220-AGGTGAGGCT-211 which is similar to $\alpha$ A-crystallin positive cis-regulatory element DE2B (-127/-118) contributing significantly to the lens expression of $\alpha \mathrm{A}$-crystallin [21].

$\gamma$-Synuclein promoter also contains nucleotide sequences similar to the regulatory elements in the promoter of another crystallin gene required for embryonic lens development- $\beta$ B1-crystallin. The motif -290 GGGGCAG-284 in the upstream region of $\gamma$-synuclein gene is similar to a part of the PL1 regulatory element in mouse $\beta \mathrm{B} 1$-crystallin, whereas the motif -274-GCTGAGCCAGCA-263 is similar to a regulatory element PL2 in $\beta$ B-crystallin gene (Figure 3) [22,23]. PL1 and PL2 sequences were identified as essential binding sites for Pax6, Maf and Prox1 transcription factors, which play a critical role in the lens development. These regulatory elements are evolutionary conserved in the promoter regions of $\beta \mathrm{B} 1$-crystallin genes. Another motives in $\gamma$-synuclein promoter similar to Pax6 binding site $[24,25]$ are the sequence -146 CTGGCCTCCGCATCAAT-130 and the sequence -12TGCACACCCACCATG including the initiating codon ATG (Figure 3).

One more feature of the $\gamma$-synuclein upstream region which makes it similar to Hsps promoters is the presence of multiple GAA/GAG or TTC/CTC motifs called heat shock elements (HSE) [26-28], for example, in the fol- 


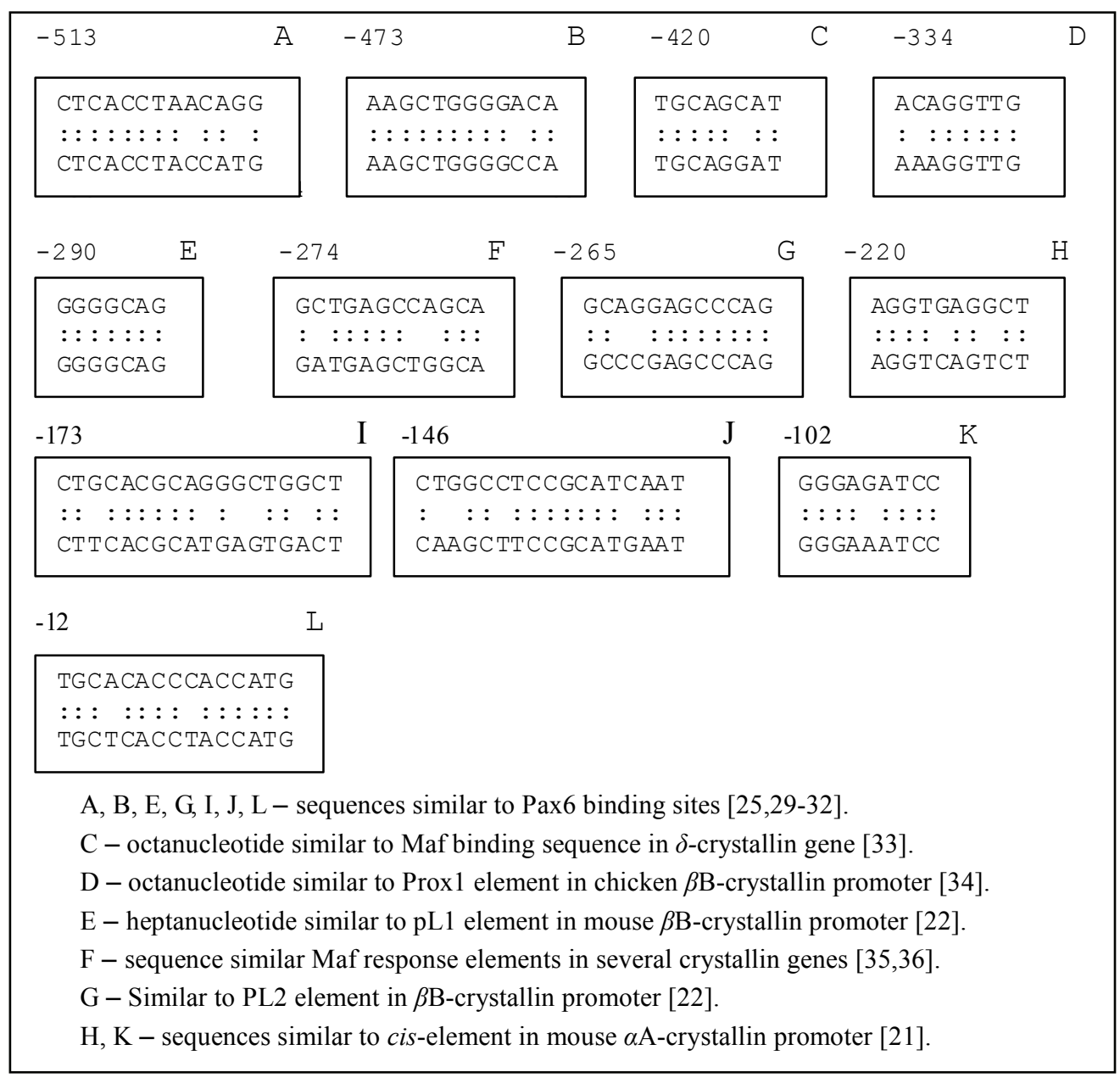

Figure 3. Comparison of the nucleotide sequences in the upstream region of $\gamma$-synuclein gene with regulatory elements of other genes implicated in the embryonic development.

lowing sequence -243CTCTGCCTTCCTATCCTGGAG GAAGGTGAGGCTGAACCTCCTTCCCTCCCTCCCT CCCTC-183.

Finally, intron 1 of $\gamma$-synuclein gene which plays a critical role in transcriptional regulation [37] contains the heptanucleotide 121-TATCAAG-127 and the motif 286-CTGAGCCTGGACGCC-300 which are identical or very close to the binding sites for Sox and Pax6 transcription factors, respectively [38].

\section{Discussion}

Currently $\alpha$-synuclein localization is studied most extensively in the brain and their expression in neurons and glial cells is described both in health and neurodegenerative diseases (see for review [8]). The role of $\gamma$-synuclein in breast cancer cells and its cellular localization is also well documented [39,40]. $\gamma$-Synuclein is also expressed in the retina and optic nerve [14]. Much less is known about localization of synucleins and their function in health and diseases in other tissues. Here we present data on localization of synucleins in the lens at different steps of embryonic development.

Lens development occurs throughout the lifetime of the individual and involves the terminal differentiation of lens epithelial cells into lens fibre cells [41]. This process begins during embryogenesis and continues into adulthood and old age.

We have found that the expression pattern of $\gamma$-synuclein in developing rat and human lens is different from that of two other members of the family. While $\alpha$-synuclein was detectable at a very low level and $\beta$-synuclein was not detectable at all, $\gamma$-synuclein was expressed on early steps of lens development, then its nuclear level is reduced during fiber cell differentiation and in mature lens $\gamma$-synuclein is not detectable.

Interestingly, previous study of three synuclein expression in embryonic and postnatal mouse development also showed that $\gamma$-synuclein has a different pattern of expression to that of two other synucleins $[12,42]$. In a search of 
a possible explanation of $\gamma$-synuclein differential expression we compared its promoter region with the sequences involved in the regulation of transcription of other lens proteins. The majority of lens proteome consists of crystallins who possess chaperonic activity and some of them play important role in the lens development. For example, $\alpha \mathrm{A}$-crystallin is a highly expressed protein in the lens which is indispensable for lens development [19,43]. Crystallin gene expression in lens is regulated at the level of transcription by a sparse number of specific DNAbinding transcription factors which bind to cis-regulatory elements in the promoter regions of their genes. Interestingly, $\gamma$-synuclein upstream region contains motives identical or similar to cis-regulatory elements in promoter regions of $\alpha \mathrm{A}$ - and $\beta \mathrm{B}$-crystallins both of which are necessary for normal lens development.

The results presented here demonstrate that $\gamma$-synuclein is expressed during a short period of normal embryonic human and rat lens formation as part of the normal development program. This suggests that it plays a role during formation of vertebrate lens. We assume that chaperonic activity of $\gamma$-synuclein is required for some of the lens embryonic pathways. Another role of $\gamma$-synuclein may be connected with its involvement in apoptotic processes, since lens fiber differentiation involved component of classical apoptosis [44]. Finally, temporarily $\gamma$-synuclein expression in embryonic lens may be associated with its ability to participate in transcriptional regulation [45] and to affect distinct growth factor-induced signaling pathways that control normal lens development. It should be noted that since $\gamma$-synuclein expression is limited to relatively short period in lens development it can be found by gene arrays analysis only if RNA samples are taken at these particular steps of embryonic development.

Interestingly, the study of synuclein expression in the mammalian cochlea showed that developmentally, $\gamma$-synuclein can be seen in the region of the outer hair cells by E19, whereas $\alpha$ - and $\beta$-synuclein do not appear at embryonic step of development [46]. These results may indicate a special role of $\gamma$-synuclein in the developmental processes of both sensory systems. Thus, we can hypothesize that during lens development synucleins may fulfill one of specific functions, e.g. chaperonic activity or play a role in the regulation of apoptosis or transcription, however, they are eliminated from mature lens because they are prone to aggregate. Indeed, as was shown by Muchowski and coauthors [47], expression of recombinant $\alpha$-synuclein in mature mouse lens leads to protein aggregation, opacification and cataract formation.

\section{ACKNOWLEDGEMENTS}

This study was supported by VA Merit Review grant and The Glau- coma Foundation grant.

\section{REFERENCES}

[1] Macario, A.J. and de Macario, E.C. (2007) Molecular chaperones: Multiple functions, pathologies, and potential applications. Frontiers in Bioscience, 12, 2588-2600.

[2] Quinlan, R. (2002) Cytoskeletal competence requires protein chaperones. Progress in Molecular and Subcellular Biology, 28, 219-233.

[3] Nagai, N., Hosokawa, M., Itohara, S., Adachi, E., Matsushita, T., Hosokawa, N., et al. (2000) Embryonic lethality of molecular chaperone Hsp47 knockout mice is associated with defects in collagen biosynthesis. Journal of Cell Biology, 150(6), 1499-1506.

[4] Dix, D.J., Allen, J.W., Collins, B.W., Mori, C., Nakamura, N., Poorman-Allen, P., et al. (1996) Targeted gene disruption of Hsp70-2 results in failed meiosis, germ cell apoptosis, and male infertility. Proceedings of the $\mathrm{Na}$ tional Academy of Sciences of the United States of America, 93(8), 3264-3268.

[5] Evans, T.G., Yamamoto, Y., Jeffery, W.R. and Krone, P.H. (2005) Zebrafish Hsp70 is required for embryonic lens formation. Cell Stress and Chaperones, 10(1), 66-78.

[6] Souza, J.M., Giasson, B.I., Leeb, V.M. and Ischiropoulosa, H. (2000) Chaperone-like activity of synucleins. FEBS Letters, 474(1), 116-119.

[7] Surgucheva, I., Ninkina, N., Buchman, V.L., Grasing, K. and Surguchov, A. (2005) Protein aggregation in retinal cells and approaches to cell protection. Cellular and Molecular Neurobiology, 25(6), 1051-1066.

[8] Surguchov, A. (2008) Molecular and cellular biology of synucleins. International Review of Cell and Molecular Biology, 270, 225-317.

[9] Perrin, R.J., Woods, W.S., Clayton, D.F. and George, J.M. (2002) Interaction of human alpha-Synuclein and Parkinson's disease variants with phospholipids. Structural analysis using site-directed mutagenesis. Journal of Biological Chemistry, 275(44), 34393-34398.

[10] Smith, D.P., Tew, D.J., Hill, A.F., Bottomley, S.P., Masters, C.L., Barnham, K.J., et al. (2008) Formation of a high affinity lipid-binding intermediate during the early aggregation phase of alpha-synuclein. Biochemistry, 47(5), 1425-1434.

[11] Ltic, S., Perovic, M., Mladenovic, A., Raicevic, N., Ruzdijic, S., Rakic, L., et al. (2004) Alpha-synuclein is expressed in different tissues during human fetal development. Journal of Molecular Neuroscience, 22(3), 199-204.

[12] Raghavan, R., Kruijff, L., Sterrenburg, M.D., Rogers, B.B., Hladik, C.L. and White, C.L. III (2004) Alphasynuclein expression in the developing human brain. $P e$ diatric and Developmental Pathology, 7(5), 506-516.

[13] Buchman, V.L., Hunter, H.J., Pinõn, L.G., Thompson, J., Privalova, E.M., Ninkina, N.N., et al. (1998) Persyn, a member of the synuclein family, has a distinct pattern of expression in the developing nervous system. Journal of Neuroscience, 18(22), 9335-9341.

[14] Surguchov, A., McMahon, B., Masliah, E. and Surgucheva, I. (2001) Synucleins in ocular tissues. Journal of Neuroscience Research, 65(1), 68-77.

[15] Blechinger, S.R., Evans, T.G., Tang, P.T., Kuwada, J.Y., 
Warren, J.T. Jr. and Krone, P.H. (2002) The heat-inducible zebrafish Hsp70 gene is expressed during normal lens development under non-stress conditions. Mechanisms of Development, 112(1-2), 213-215.

[16] Boyle, D.L., Takemoto, L., Brady, J.P. and Wawrousek, E.F. (2003) Morphological characterization of the Alpha A- and Alpha B-crystallin double knockout mouse lens. BMC Ophthalmology, 3(3), 1-11.

[17] Tallot, P., Grongnet, J.F. and David, J.C. (2003) Dual perinatal and developmental expression of the small heat shock proteins aB-crystallin and Hsp27 in different tissues of the developing piglet. Biology of the Neonate, 83(4), 281-288

[18] Chenna, R., Sugawara, H., Koike, T., Lopez, R., Gibson, T.J., Higgins, D.G., et al. (2003) Multiple sequence alignment with the Clustal series of programs. Nucleic Acids Research, 31(13), 3497-3500.

[19] Nakamura, T., Donovan, D.M., Hamada, K., Sax, C.M., Norman, B., Flanagan, J.R., et al. (1990) Regulation of the mouse alpha A-crystallin gene: Isolation of a cDNA encoding a protein that binds to a cis sequence motif shared with the major histocompatibility complex class I gene and other genes. Molecular and Cellular Biology, 10(7), 3700-3708.

[20] Jaworski, C.J., Chepelinsky, A.B. and Piatigorsky, J. (1991) The alpha A-crystallin gene: Conserved features of the 5 '-flanking regions in human, mouse, and chicken. Journal of Molecular Evolution, 33(6), 495-505.

[21] Klement, J.F., Cvekl, A. and Piatigorsky, J. (1993) Functional elements DE2A, DE2B, and DE1A and the TATA box are required for activity of the chicken alpha A-crystallin gene in transfected lens epithelial cells. Journal of Biological Chemistry, 268, 6777-6784.

[22] Mizuno, N., Ueda, Y. and Kondoh, H. (2005) Requirement for betaB1-crystallin promoter of Xenopus laevis in embryonic lens development and lens regeneration. Development, Growth and Differentiation, 47(3), 131-140.

[23] Cui, W., Tomarev, S.I., Piatigorsky, J., Chepelinsky, A.B. and Duncan, M.K. (2004) Mafs, Prox1, and Pax6 can regulate chicken betaB1-crystallin gene expression. Journal of Biological Chemistry, 279(12), 11088-11095.

[24] Epstein, J., Cai, J., Glaser, T., Jepeal, L. and Maas, R. (1994) Identification of a Pax paired domain recognition sequence and evidence for DNA-dependent conformational changes. Journal of Biological Chemistry, 269(11), 8355-8361.

[25] Zhou, Y.H., Zheng, J.B., Gu, X., Saunders, G.F. and Yung, W.K. (2002) Novel Pax6 binding sites in the human genome and the role of repetitive elements in the evolution of gene regulation. Genome Research, 12(11), 1716-1722.

[26] Amin, J., Ananthan, J. and Voellmy, R. (1988) Key features of heat shock regulatory elements. Molecular and Cellular Biology, 8(9), 3761-3769.

[27] Perisic, O., Xiao, H. and Lis, J.T. (1989) Stable binding of Drosophila heat shock factor to head-to-head and tail-to-tail repeats of a conserved 5 bp recognition unit. Cell, 59(5), 797-806

[28] Yasuda, K., Ishihara, K., Nakashima, K. and Hatayama, T. (1999) Genomic cloning and promoter analysis of the mouse 105-kDa heat shock protein (HSP105) gene. Biochemical and Biophysical Research Communications, 256(1), 75-80.
[29] Van Heyningen, V. and Williamson, K.A. (2002) Pax6 in sensory development. Human Molecular Genetics, 11(10), 1161-1167.

[30] Kamachi, Y., Uchikawa, M., Tanouchi, A., Sekido, R. and Kondoh, H. (2001) Pax6 and SOX2 form a co-DNAbinding partner complex that regulates initiation of lens development. Genes and Development, 15(10), 272-286.

[31] Lengler, J., Bittner, T., Münster, D., Gawad, A.-D. and Graw, J. (2005) Agonistic and antagonistic action of AP2, Msx2, Pax6, Prox1 AND Six3 in the regulation of Sox2 expression. Ophthalmic Research, 37(6), 301-309.

[32] Yang, Y., Stopka, T., Golestaneh, N., Wang, Y., Wu, K., Li, A., et al. (2006) Regulation of alphaA-crystallin via Pax6, c-Maf, CREB and a broad domain of lens-specific chromatin. EMBO Journal, 25(10), 2107-2118.

[33] Muta, M., Kamachi, Y., Yoshimoto, A., Higashi, Y. and Kondoh, H. (2002) Distinct roles of SOX2, Pax6 and Maf transcription factors in the regulation of lens-specific delta1-crystallin enhancer. Genes to Cells, 7(8), 791-805.

[34] Chen, X., Taube, J.R., Simirskii, V.I., Patel, T.P. and Duncan, M.K. (2008) Dual roles for Prox1 in the regulation of the chicken betaB1-crystallin promoter. Investigative Ophthalmology \& Visual Science, 49(4), 1542-1552.

[35] Kerppola, T.K. and Curran, T. (1994) Maf and Nrl can bind to AP-1 sites and form heterodimers with Fos and Jun. Oncogene, 9(3), 675-684.

[36] Mahoney, K.M., Petrovic, N., Schacke, W. and Shapiro, L.H. (2007) CD13/APN transcription is regulated by the proto-oncogene c-Maf via an atypical response element. Gene, 403(1-2), 178-187.

[37] Lu, A., Gupta, A., Li, C., Ahlborn, T.E., Ma, Y., Shi, E.Y., et al. (2001) Molecular mechanisms for aberrant expression of the human breast cancer specific gene 1 in breast cancer cells: control of transcription by DNA methylation and intronic sequences. Oncogene, 20(37), 5173-5185.

[38] Grinchuk, O., Kozmik, Z., Wu, X. and Tomarev, S. (2005) The Optimedin gene is a downstream target of Pax6. Journal of Biological Chemistry, 280(10), 35228-35237.

[39] Jia, T., Liu, Y.E., Liu, J. and Shi, Y.E. (1999) Stimulation of breast cancer invasion and metastasis by synuclein gamma. Cancer Research, 59(3), 742-747.

[40] Gupta, A., Inaba, S., Wong, O.K., Fang, G. and Liu, J. (2003) Breast cancer-specific gene 1 interacts with the mitotic checkpoint kinase BubR1. Oncogene, 22(48), 7593-7599.

[41] Wride, M.A. (1996) Cellular and molecular features of lens differentiation: a review of recent advances. Differentiation, 61(2), 77-93.

[42] Ninkina, N.N., Privalova, E.M., Pinon, L.G., Davies, A.M. and Buchman, V.L. (1999) Developmentally regulated expression of persyn, a member of the synuclein family, in skin. Experimental Cell Research, 246(2), 308311.

[43] Brady, J.P., Garland, D.L., Green, D.E., Tamm, E.R., Giblin, F.J. and Wawrousek, E.F. (2001) AlphaB-crystallin in lens development and muscle integrity: A gene knockout approach. Investigative Ophthalmology \& Visual Science, 42(12), 2924-2934

[44] Wride, M.A. (2000) Minireview: Apoptosis as seen through a lens. Apoptosis, 5(3), 203-209.

[45] Surgucheva, I. and Surguchov, A. (2008) $\gamma$-synuclein: Cell-type-specific promoter activity and binding to tran- 
scription factors. Journal of Molecular Neuroscience, 35(3), 267-271.

[46] Akil, O., Weber, C.M., Park, S.N., Ninkina, N.N., Buchman, V.L. and Lustig, L.R. (2008) Localization of synucleins in the mammalian cochlea. Journal of the Association for Research in Otolaryngology, 9(4), 452-463.
[47] Muchowski, P.J., Ramsden, R., Nguyen, Q., Arnett, E.E., Greiling, T.M., Anderson, S.K., et al. (2008) Noninvasive measurement of protein aggregation by mutant huntingtin fragments or alpha-synuclein in the lens. Journal of Biological Chemistry, 283(10), 6330-6336. 